\title{
CONTROL NULO PARA LA ECUACIÓN LINEAL DEL CALOR SOBRE ESPACIOS CON PESO
}

\author{
Victor Cabanillas Zannini ${ }^{1}$, Yolanda Santiago Ayala ${ }^{2}$.
}

(Recibido: 29/08/2014 - Aceptado: 03/09/2014)

Resumen: En este trabajo estudiaremos la controlabilidad nula de la ecuación lineal del calor sobre espacios de Lebesgue y de Sobolev con peso. Dado el PVIF

$$
\begin{cases}\frac{\partial y}{\partial t}-\Delta y+a y+b . \nabla y=g & , \text { en } Q=\Omega \times(0, T) \\ y=0 & , \text { sobre } \Sigma=\Gamma \times(0, T) \\ y(0)=y_{0} & , \text { en } \Omega\end{cases}
$$

demostraremos la existencia de un control $u$ en espacios con peso que al actuar en el interior del dominio, conduce al sistema al equilibrio. La herramienta que usaremos para tal fin son las Desigualdades de Carleman.

Palabras Claves: Controlabilidad nula, control interno, desigualdad de Carleman, espacios con peso.

\section{NULL CONTROLLABILITY OF THE LINEAR HEAT EQUATION ON THE WEIGHT SPACES}

Abstract: In this work, we study the null controllability of the linear heat equation on Lebesgue and Sobolev weight spaces. Given the initial and boundary value problem

$$
\begin{cases}\frac{\partial y}{\partial t}-\Delta y+a y+b . \nabla y=g & , \text { en } Q=\Omega \times(0, T) \\ y=0 & , \text { sobre } \Sigma=\Gamma \times(0, T) \\ y(0)=y_{0} & , \text { en } \Omega\end{cases}
$$

we show the existence of a control function $u$ belong to weight spaces that to acts on a subset of the domain $\Omega$ leads system to the equilibrium. The principal tool that we use is the Carleman's inequality.

Key Words: Null controllability, internal control, Carleman inequality, weight spaces.

\section{Introducción}

Sea $\Omega$ un dominio acotado de $\mathbb{R}^{n}, n \geq 1$, con frontera $\Gamma=\partial \Omega$ de clase $C^{2}$. Consideremos la ecuación del calor

$$
\begin{cases}\frac{\partial y}{\partial t}-\Delta y+a y+b . \nabla y=g & , \text { en } Q=\Omega \times(0, T) \\ y=0 & , \text { sobre } \Sigma=\Gamma \times(0, T) \\ y(0)=y_{0} & , \text { en } \Omega\end{cases}
$$

\footnotetext{
${ }^{1}$ UNMSM, Facultad de Ciencias Matemáticas, e-mail: vcabanillasz@unmsm.edu.pe

${ }^{2}$ UNMSM, Facultad de Ciencias Matemáticas, e-mail:ysantiagoa@unmsm.edu.pe
} 
donde $a$ y $b$ son potenciales que pertenecen a $L^{\infty}(Q)$ y $\left(L^{\infty}(Q)\right)^{n}$ respectivamente.

En [2], L.A. Fernández y E. Zuazua demostraron que el sistema (1) es aproximadamente controlable en $L^{2}(\Omega)$ usando el enfoque de los problemas de control optimal. Como la controlabilidad nula de (1) implica la controlabilidad aproximada de dicho sistema, es natural estudiar la controlabilidad nula de (1).

\section{Formulación del problema}

El problema de la controlabilidad nula para el sistema (1) puede ser formulado de la siguiente manera: dados $T>0, y_{0}$, y $f$, hallar un par $(y, u)$ que satisfaga

$$
\begin{cases}\frac{\partial y}{\partial t}-\Delta y+a y+b . \nabla y=g+u \chi_{\omega} & , \text { en } Q=\Omega \times(0, T) \\ y=0 & , \text { sobre } \Sigma=\Gamma \times(0, T) \\ y(0)=y_{0} & , \text { en } \Omega\end{cases}
$$

y tal que

$$
y(T, x)=0, \quad x \in \Omega .
$$

En (2), la función $u=u(x, t)$ es la función control y la función característica $\chi_{\omega}$ indica que este control actúa únicamente sobre el cilindro $Q_{\omega}=\omega \times(0, T)$, siendo $\omega$ un subdominio arbitrario y no vacío de $\Omega$.

El problema (2)- (3) fue estudiado por A. Fursikov y O. Yu. Imanuvilov en [5] con una hipótesis de mayor regularidad sobre el potencial $b$, más precisamente, ellos consideraron $b_{i} \in C^{0,1}(\bar{Q})$ y utilizaron las desigualdades de Carleman en norma $L^{2}(\Omega)$ para ecuaciones parabólicas. Cuando el potencial $b_{i}$ pertenece a $L^{\infty}(Q)$ tales desigualdades no pueden ser utilizadas para concluir la controlabilidad nula del problema (2) - (3). En este caso se hace necesario el uso de desigualdades de Carleman con estimativas en norma $H^{-1}(\Omega)$.

En [6], O. Yu. Imanuvilov y M. Yamamoto introdujeron desigualdades de Carleman que envuelven normas en $H^{-s}(\Omega), s \in[0,1]$ y demostraron cómo tales desgualdades pueden ser empleadas en el estudio del control exacto distribuido de un sistema parabólico general.

En este trabajo usaremos las desigualdades de Carleman de O. Yu. Imanuvilov y $\mathrm{M}$. Yamamoto para demostrar la controlabilidad nula de (2)- (3). Además, el trabajo muestra la manera como construir el control que conduce la solución al equilibrio después de un tiempo $T$.

\section{Desigualdad de Carleman}

Consideremos el problema de valor inicial y frontera

$$
\begin{cases}L y=\frac{\partial y}{\partial t}-\Delta y+a y+b \cdot \nabla y=g & , \text { en } Q=\Omega \times(0, T) \\ y=0 & , \text { sobre } \Sigma=\Gamma \times(0, T) \\ y(0)=v_{0} & , \text { en } \Omega\end{cases}
$$

donde $a \in L^{\infty}(Q), b \in\left(L^{\infty}(Q)\right)^{n}$ y $y_{0}, g$ son funciones dadas.

Lema 3.1 Sea $\omega_{0} \Subset \omega$ un subdominio arbitrario de $\Omega$. Entonces existe una función $\psi \in C^{2}(\bar{\Omega})$ tal que

$$
\left\{\begin{array}{l}
\psi(x)>0 \text { para todo } x \in \Omega,\left.\quad \psi\right|_{\Gamma}=0 \\
|\nabla \psi(x)|>0 \text { para todo } x \in \overline{\Omega \backslash \omega_{0}}
\end{array}\right.
$$

Demostración. Ver [5]. 
Usando la función $\psi$ del lema anterior definimos el peso

$$
\varphi(x)=\frac{e^{\lambda \psi(x)}}{t(T-t)}
$$

y las funciones

$$
\alpha(t, x)=\frac{e^{\lambda \psi(x)}-e^{2 \lambda\|\psi\|_{C(\bar{\Omega})}}}{t(T-t)} \quad \mathrm{y} \quad \eta(t, x)=\frac{-e^{\lambda \psi(x)}+e^{2 \lambda\|\psi\|_{C(\bar{\Omega})}}}{(T-t) l(t)}
$$

donde $l \in C^{\infty}([0, T]), l(t)>0, l(t) \geq t, \forall t \in[0, T], l(t)=t, \forall t \in\left[\frac{T}{2}, T\right]$.

Denotemos por $L^{*}$ el operador adjunto formal de $L$. Definimos a continuación la solución débil del problema (4) usando el Método de Transposición.

Definición 3.2 Diremos que la función $y \in L^{2}(Q)$ es solución del problema (4) si

$$
\int_{Q} y L^{*} z d x d t=\int_{Q} g z d x d t+\int_{\Omega} v_{0} z(0) d x
$$

para todo z perteneciente al siguiente espacio de prueba.

$$
\left\{z \in L^{2}\left(0, T ; H^{1}(\Omega)\right) ; L^{*} z \in L^{2}(Q),\left.z\right|_{\partial \Omega}=0, z(T)=0\right\} .
$$

Teorema 3.3 (Desigualdad de Carleman) Sean $a \in L^{\infty}(Q), b \in\left(L^{\infty}(Q)\right)^{n}$ y las funciones $\varphi$ y $\alpha$ definidas en (6) y (7). Supongamos además que la función g se puede expresar como

$$
g(t, x)=g_{0}(t, x)+\sum_{1 \leq i \leq n} \frac{\partial g_{i}}{\partial x_{i}}(t, x)
$$

con $g_{i} \in L^{2}(Q), 0 \leq i \leq n$. Entonces existe un número $\widehat{\lambda}>0$ tal que para $\lambda>\hat{\lambda}$ arbitrario es posible encontrar un número $s_{0}(\lambda)>0$ satisfaciendo la siguiente condición: existe una constante $C_{1}>0$ tal que para cada $s \geq s_{0}(\lambda)$, la solución $y \in L^{2}(Q)$ del problema (4) satisface la desigualdad

$$
\begin{gathered}
\int_{Q}\left(\frac{1}{s \varphi}|\nabla y|^{2}+s \varphi|y|^{2}\right) e^{2 s \alpha} d x d t \leq \\
\leq C_{1}\left(\left\|g_{0} e^{s \alpha}\right\|_{L^{2}\left(0, T ; H^{-1}(\Omega)\right)}^{2}+\sum_{1 \leq i \leq n}\left\|g_{i} e^{s \alpha}\right\|_{L^{2}(Q)}^{2}+\int_{Q_{\omega}}(s \varphi)|y|^{2} e^{2 s \alpha} d x d t\right)
\end{gathered}
$$

donde la constante $C_{1}$ depende continuamente de $\lambda,\|a\|_{L^{\infty}(Q)} y\|b\|_{\left(L^{\infty}(Q)\right)^{n}}$, y es independiente de $s$.

Demostración. Ver [6].

A continuación introducimos el espacio $L^{2}(Q)$ con peso $(T-t) e^{2 s \eta}$, al que denotaremos por $X_{s}^{\lambda}(Q)$, es decir,

$$
X_{s}^{\lambda}(Q)=L^{2}\left(Q,(T-t) e^{2 s \eta}\right) .
$$

Con este espacio definimos

$$
Z_{s}^{\lambda}(Q)=\left\{y(t, x) ;\left.y\right|_{\partial \Omega \times(0, T)}=0, \nabla y \in L^{2}\left(Q, e^{2 s \eta}\right), L y \in X_{s}^{\lambda}(Q)\right\}
$$

sobre el cual podemos definir la norma

$$
\|y\|_{Z_{s}^{\lambda}(Q)}^{2}=\|L y\|_{X_{s}^{\lambda}(Q)}^{2}+\|y\|_{L^{2}\left(Q, e^{2 s \eta}\right)}^{2}+\|\nabla y\|_{L^{2}\left(Q, e^{2 s \eta}\right)}^{2}
$$

Por último, el espacio

con norma

$$
Y(Q)=\left\{y(t, x) ;\left.y\right|_{\partial \Omega \times(0, T)}=0, L y \in L^{2}(Q), y(0) \in H^{1}(\Omega)\right\}
$$

$$
\|y\|_{Y(Q)}^{2}=\|L y\|_{L^{2}(Q)}^{2}+\|y(0)\|_{H^{1}(Q)}^{2} .
$$




\section{Controlabilidad nula interna}

En las condiciones anteriores, consideremos el PVIF asociado a la ecuación del calor

$$
\begin{cases}L y=\frac{\partial y}{\partial t}-\Delta y+a y+b . \nabla y=g+u & , \text { en } Q=\Omega \times(0, T) \\ y=0 & , \text { sobre } \Sigma=\Gamma \times(0, T) \\ y(0)=v_{0} & , \text { en } \Omega\end{cases}
$$

donde $T>0, v_{0}$ y $g$ son funciones dadas y la función $u=u(t, x)$ es la función control actuando al interior del dominio $\Omega$. En relación a la función control $u$, supondremos que pertenece al espacio $\mathcal{U}(\omega)$ definido por

$$
\mathcal{U}(\omega)=\left\{u=u(t, x) \in L^{2}(Q) ; \operatorname{sop}(u) \subset \overline{Q_{\omega}}=\bar{\omega} \times[0, T]\right\}
$$

Definición 4.1 Diremos que el sistema (9) es nulamente controlable si, dado $T>0$, existe un control $u \in \mathcal{U}(\omega)$ tal que la solución y de (9) alcanza el estado de equilibrio transcurrido el tiempo $T$, es decir

$$
y(T, x)=0, \text { en } \Omega .
$$

A continuación enunciamos y demostramos nuestro resultado central.

Teorema 4.2 Sean $\lambda>\hat{\lambda} y v_{0} \in H_{0}^{1}(\Omega)$. En las condiciones anteriores, existe una constante $s_{0}(\lambda)$ tal que si $g \in X_{s}^{\lambda}(Q)$ con $s \geq s_{0}(\lambda)$, entonces el problema (9) tiene una solución $(y, u)$ en la clase

$$
(y, u) \in\left(Y(Q) \cap Z_{s}^{\lambda}(Q)\right) \times\left(\mathcal{U}(\omega) \cap X_{s}^{\lambda}(Q)\right)
$$

que verifica (11), es decir, el sistema (9) es nulamente controlable. Además, la solución y de (9) depende continuamente del dato inicial y el término fuente g. Más precisamente, existe una constante $C(\lambda, s)>0$ tal que

$$
\|y\|_{\left(Y(Q) \cap Z_{s}^{\lambda}(Q)\right) \times\left(\mathcal{U}(\omega) \cap X_{s}^{\lambda}(Q)\right)} \leq C(\lambda, s)\left[\left\|v_{0}\right\|_{H_{0}^{1}(\Omega)}+\|g\|_{X_{s}^{\lambda}(Q)}\right] .
$$

Demostración. El número $\widehat{\lambda}$ es el mismo del Teorema (3.3). Entonces, para cada $\lambda>\widehat{\lambda}$ existe una constante $s_{0}(\lambda)$ tal que si $s \geq s_{0}(\lambda)$, la desigualdad de Carleman (8) es válida.

Consideremos la sucesión $\left(\rho_{k}\right)_{k}$ de pesos definida por

$$
\rho_{k}(t, x)=\exp \left(\frac{2 s \eta(t, x)(T-t)}{T-t+\frac{1}{k}}\right)
$$

y la sucesión

$$
m_{k}(x)=\left\{\begin{array}{llc}
1 & , \quad x \in \bar{\omega} \\
k & , \quad x \in \Omega-\bar{\omega}
\end{array}\right.
$$

Entonces definimos el funcional

$$
J_{k}: Z_{s}^{\lambda}(Q) \times X_{s}^{\lambda}(Q) \rightarrow \mathbb{R}
$$

por

$$
J_{k}(y, u)=\frac{1}{2} \int_{Q} \rho_{k}\left(|\nabla y|^{2}+|y|^{2}\right) d x d t+\frac{1}{2} \int_{Q}(T-t) e^{2 s \eta} m_{k} u^{2} d x d t
$$

y consideremos el problema variacional

$$
\operatorname{linf}_{(y, u) \in \Lambda} J_{k}(y, u)
$$

donde

$$
\Lambda=\left\{(y, u) \in Z_{s}^{\lambda}(Q) \times X_{s}^{\lambda}(Q) ;(y, u) \text { satisface (9) y (11) }\right\}
$$


Siguiendo las ideas de C. Fabre, J.P. Puel y E. Zuazua [1] se puede demostrar que el funcional $J_{k}$ es continuo, convexo y coercivo y gracias a un resultado de continuación única, consecuencia de la desigualdad de Carleman (8), se demuestra que $J_{k}$ es estrictamente convexo. Entonces, siguiendo los métodos expuestos en [8] se concluye que el sistema (14) admite una única solución $\left(\widehat{y}_{k}, \widehat{u}_{k}\right) \in Y(Q) \cap L^{2}(Q)$.

La ecuación de Euler-Lagrange para la solución $\left(\widehat{y}_{k}, \widehat{u}_{k}\right)$ de $(14)$ es dada por

$$
\int_{Q}\left(-\nabla \cdot\left(\rho_{k} \nabla \widehat{y}_{k}\right)+\rho_{k} \widehat{y}_{k}\right) y d x d t+\int_{Q}(T-t) e^{2 s \eta} m_{k} \widehat{u}_{k} u d x d t=0
$$

para todo $(y, u) \in \Lambda$.

Siguiendo [8], podemos hallar el siguiente sistema de optimalidad

$$
\begin{aligned}
& \begin{cases}L \widehat{y}_{k}=\frac{\partial \widehat{y}_{k}}{\partial t}-\Delta \widehat{y}_{k}+a \widehat{y}_{k}+b \cdot \nabla \widehat{y}_{k}=g+\widehat{u}_{k} & , \\
\left.\widehat{y}_{k}\right|_{\Sigma}=0, \quad \widehat{y}_{k}(0)=v_{0}, \quad \widehat{y}_{k}(T)=0 & \text { en } Q\end{cases} \\
& \begin{cases}L^{*} p_{k}=-\frac{\partial p_{k}}{\partial t}-\Delta p_{k}+a p_{k}-\operatorname{div}\left(b p_{k}\right)=\nabla \cdot\left(\rho_{k} \nabla \widehat{y}_{k}\right)-\rho_{k} \widehat{y}_{k} & , \text { en } Q \\
\left.p_{k}\right|_{\Sigma}=0, \quad p_{k}=(T-t) e^{2 s \eta} m_{k} \widehat{u}_{k} & \text { en } \Omega\end{cases}
\end{aligned}
$$

Aplicando la desigualdad de Carleman (8) a (16) podemos demostrar la siguiente desigualdad:

Proposición 4.3 Con la notación y condiciones anteriores tenemos

$$
\begin{aligned}
\int_{Q} e^{-2 s \eta} \frac{\left|p_{k}\right|^{2}}{T-t} d x d t+\int_{\Omega}\left|p_{k}(0, x)\right|^{2} d x & \leq C(\lambda, s)\left[\int_{Q} \rho_{k}^{2} e^{-2 s \eta}\left(\left|\nabla \widehat{y}_{k}\right|^{2}+\left|\widehat{y}_{k}\right|^{2}\right) d x d t+\right. \\
& \left.+\int_{Q_{\omega}} e^{-2 s \eta} \frac{\left|p_{k}\right|^{2}}{T-t} d x d t\right]
\end{aligned}
$$

Continuación de la demostración del Teorema (4.2) Gracias a esta proposición y teniendo en cuenta que $\left|\rho_{k} e^{-2 s \eta}\right| \leq 1$ y (16) se sigue que

$$
\begin{aligned}
\int_{Q} e^{-2 s \eta} \frac{\left|p_{k}\right|^{2}}{T-t} d x d t+\int_{\Omega}\left|p_{k}(0, x)\right|^{2} d x & \leq C(\lambda, s)\left[\int_{Q} \rho_{k}\left(\left|\nabla \widehat{y}_{k}\right|^{2}+\left|\widehat{y}_{k}\right|^{2}\right) d x d t\right. \\
& \left.+\int_{Q_{\omega}} e^{2 s \eta}(T-t) d x d t\right]
\end{aligned}
$$

Efectuando el producto $L^{2}(Q)$ de la ecuación (16 ) por $\widehat{y}_{k}$ e integrando por partes tenemos

$$
\begin{gathered}
\int_{Q} \rho_{k}\left(\left|\nabla \widehat{y}_{k}\right|^{2}+\left|\widehat{y}_{k}\right|^{2}\right) d x d t+\int_{Q} e^{2 s \eta}(T-t) m_{k}\left|\widehat{u}_{k}\right|^{2} d x d t+ \\
+\int_{Q} g p_{k} d x d t+\left(p_{k}(0), v_{0}\right)_{L^{2}(\Omega)}=0
\end{gathered}
$$

Además, de la definición del funcional $J_{k}$ tenemos

$$
J_{k}\left(\widehat{y}_{k}, \widehat{u}_{k}\right)=-\frac{1}{2} \int_{Q} g p_{k} d x d t-\frac{1}{2}\left(p_{k}(0), v_{0}\right)_{L^{2}(\Omega)}
$$

Luego

$$
J_{k}\left(\widehat{y}_{k}, \widehat{u}_{k}\right) \leq \frac{1}{2}\|g\|_{X_{s}^{\lambda}(Q)}\left(\int_{Q} \frac{\left|p_{k}\right|^{2}}{T-t} d x d t\right)^{\frac{1}{2}}+\frac{1}{2}\left\|p_{k}(0)\right\|_{L^{2}(\Omega)}\left\|v_{0}\right\|_{L^{2}(\Omega)}
$$


Ahora, aplicando (17) en (21) llegamos a

$$
J_{k}\left(\widehat{y}_{k}, \widehat{u}_{k}\right) \leq C^{2}(\lambda, s)\left(\|g\|_{X_{s}^{\lambda}(Q)}+\left\|v_{0}\right\|_{L^{2}(\Omega)}\right)^{2}
$$

De esta desigualdad y de la definición del funcional $J_{k}$ tenemos que existe una subsucesión de $\left\{\left(\widehat{y}_{k}, \widehat{u}_{k}\right)\right\}_{k \in \mathbb{N}}$, para la cual usaremos la misma notación, tal que

$$
\begin{aligned}
& \widehat{y}_{k} \rightarrow y \quad \text { débilmente en } Y(Q) \\
& \widehat{u}_{k} \rightarrow u \quad \text { débilmente en } L^{2}(Q)
\end{aligned}
$$

Usando estas dos convergencias podemos pasar al límite en (16) y comprobar que el par $(y, u)$ es solución del sistema (9) y que y satisface (11).

De la desigualdad (22) podemos demostrar que

$$
\widehat{u}_{k} \rightarrow 0 \text { fuertemente en } L^{2}\left(Q-Q_{\omega}\right)
$$

g

$$
e^{s \eta} \widehat{u}_{k} \rightarrow e^{s \eta} u \quad \text { débilmente en } L^{2}\left(Q_{\omega}\right)
$$

Si denotamos por $Q_{\varepsilon}=\Omega \times(0, T-\varepsilon)$, también se prueba que

$$
\left(\sqrt{\rho_{k}} \frac{\partial \widehat{y}_{k}}{\partial x_{i}}, \sqrt{\rho_{k}} \widehat{y}_{k}\right) \rightarrow\left(e^{s \eta} \frac{\partial y}{\partial x_{i}}, e^{s \eta} y\right) \text { débilmente en } L^{2}\left(Q_{\varepsilon}\right), \forall \varepsilon>0
$$

Estas últimas tres convergencias permiten mostrar que $(y, u)$ pertenece a la clase (12), es decir,

$$
y \in Y(Q) \cap Z_{s}^{\lambda}(Q) \quad \text { y } \quad u \in \mathcal{U}(\omega) \cap X_{s}^{\lambda}(Q) .
$$

Por último, la dependencia continua (13) es una consecuencia de (22), (23), (24), las convergencias anteriores y el lema de Fatou. 


\section{REFERENCIAS BIBLIOGRÁFICAS}

[1] C. Fabre, J. P. Puel y E. Zuazua, Approximate controllability of the semilinear heat equation. Proc. Royal Soc. Edinburgh, 125A, (1995), pp. 31-61.

[2] L. A. Fernández y E. Zuazua, Approximate controllability for the semilinear heat equation involving gradient terms. J. Optim. Theory and Appl. Vol. 101, $\mathrm{N}^{0}$ 2, (1999), pp. 307-328.

[3] E. Fernández-CARA, Null controllability of the semilinear heat equation. ESAIM: COCV, Vol 2, (1997), pp. 87-103. URL: http://www.emath.fr/cocv/

[4] A. Fursikov, Optimal Control of Distributed Systems. Theory and Applications. Transl. of Math. Monographs. Vol 187, A.M.S. (2000).

[5] A. Fursikov y O. Yu. Imanuvilov, Controllability of evolution equations. Lecture Notes Series \#34, Research Institute of Mathematics, Global Analysis Research Center, Seoul National University, (1996).

[6] O. Yu. Imanuvilov y M. Yamamoto, On Carleman inequalities for parabolic equations in Sobolev spaces of negative order and exact controllability for semilinear parabolic equations. Preprint \# 98-46, University of Tokyo. Graduate School of Mathematics, Tokyo, Japan, (1998).

[7] G. Lebeau y L. Robbiano, Contrôle exact de l'equation de la chaleur. Comm. P.D.E., 20 (1995), pp. 335-356.

[8] J. L. Lions, Contrôle des Systèmes Distribués Singuliers, Gauthier-Villars, Paris, (1983).

[9] J. L. Lions, Quelques Méthodes de resolution des problèmes aux limites non linéaires. Gauthier-Villars, Paris (1969).

[10] E. ZuAzuA, Approximate controllability for semilinear heat equation: boundary control. Computational Science for the 21st Century, M. O. Bristeau et al. eds., John Wiley \& Sons, (1997), pp. 738-747.

[11] E. ZuazuA, Some problems and results on the controllability of Partial Differential Equations. Proceedings of the Second European Conference of Mathematics, Budapest,(1996). Progress in Mathematics 169, (1998), pp. 276-311. 